Reduction of Large Detailed Chemical Kinetic Mechanisms for Autoignition Using Joint Analyses of Reaction Rates and Sensitivities

A. Saylam, M. Ribaucour, W. J. Pitz, R. Minetti

December 18, 2006

International Journal of Chemical Kinetics 
This document was prepared as an account of work sponsored by an agency of the United States Government. Neither the United States Government nor the University of California nor any of their employees, makes any warranty, express or implied, or assumes any legal liability or responsibility for the accuracy, completeness, or usefulness of any information, apparatus, product, or process disclosed, or represents that its use would not infringe privately owned rights. Reference herein to any specific commercial product, process, or service by trade name, trademark, manufacturer, or otherwise, does not necessarily constitute or imply its endorsement, recommendation, or favoring by the United States Government or the University of California. The views and opinions of authors expressed herein do not necessarily state or reflect those of the United States Government or the University of California, and shall not be used for advertising or product endorsement purposes. 


\title{
Reduction of large detailed chemical kinetic mechanisms for autoignition using joint analyses of reaction rates and sensitivities
}

\author{
A. Saylam*, M. Ribaucour", W. J. Pitz ${ }^{\text {}}$, and R. Minetti* \\ PhysicoChimie des Processus de Combustion et de l'Atmosphère, UMR CNRS/ 8522 \\ \#Université des Sciences et Technologies de Lille, 59655 Villeneuve d'Ascq cedex, France \\ *Université d'Artois, LCPA, Porte Nord, 62700 Bruay-la-Buissière, France \\ "Lawrence Livermore National Laboratory, Livermore, CA 94551 USA
}

\# corresponding author 


\section{Nomenclature list}

\begin{tabular}{|c|c|}
\hline symbol & name \\
\hline$A_{\mathrm{i}}$ & pre-exponential factor of the reaction $\mathrm{i}$ \\
\hline$E$ & Average error on the ignition delay time \\
\hline$\varepsilon_{\mathrm{r}}$ & rate-threshold \\
\hline FM & full mechanism \\
\hline$G_{\text {fast }}$ & group of fast reactions \\
\hline$G_{r-1}$ & group of rate-limiting reactions \\
\hline$k$ & index assigned to a reaction i according to the magnitude of $\overline{S_{\mathrm{OH}, \mathrm{i}}}$ \\
\hline$k_{\text {cut }}$ & cutting rank \\
\hline$N_{\mathrm{r}}$ & number of reactions in the full mechanism \\
\hline$N_{r-1}$ & number of rate-limiting reactions \\
\hline$N_{\mathrm{s}}$ & number of species in the full mechanism \\
\hline$r_{\mathrm{i}}$ & net rate of the reaction $\mathrm{i}$ \\
\hline$\overline{r_{\mathrm{i}}(t)}$ & normalized rate of the reaction i at the time $t$ \\
\hline RM & reduced mechanism \\
\hline$S_{\mathrm{j}, \mathrm{i}}$ & first-order local sensitivity coefficient of the species $j$ to the reaction $i$ \\
\hline$\overline{S_{j, i}}$ & log-normalized sensitivity coefficient of the species $\mathrm{j}$ to the reaction $\mathrm{i}$ \\
\hline$\overline{S_{\mathrm{OH}, \mathrm{i}}}$ & log-normalized sensitivity coefficient of $\mathrm{OH}$ radical to the reaction $\mathrm{i}$ \\
\hline$\overline{S_{\mathrm{OH}, \mathrm{k}}}$ & $\begin{array}{l}\text { log-normalized sensitivity coefficient of } \mathrm{OH} \text { radical to the reaction in } \\
\mathrm{k}^{\text {th }} \text { position in the rank of coefficients } \overline{S_{\mathrm{OH}, \mathrm{i}}} \text { in decreasing order }\end{array}$ \\
\hline$t^{*}$ & time at which the analysis of sensitivity is performed \\
\hline$T_{\text {ana }}$ & analysis temperature \\
\hline$t_{\text {ign }}$ & total ignition time \\
\hline$Z_{\mathrm{j}}(t)$ & mass fraction of the species $\mathrm{j}$ a the time $t$ \\
\hline
\end{tabular}




\section{Abstract}

A new technique of reduction of detailed mechanisms for autoignition, which is based on two analysis methods is described. An analysis of reaction rates is coupled to an analysis of reaction sensitivity for the detection of redundant reactions. Thresholds associated with the two analyses have a great influence on the size and efficiency of the reduced mechanism. Rules of selection of the thresholds are defined. The reduction technique has been successfully applied to detailed autoignition mechanisms of two reference hydrocarbons: $n$-heptane and iso-octane. The efficiency of the technique and the ability of the reduced mechanisms to reproduce well the results generated by the full mechanism are discussed. A speedup of calculations by a factor of 5.9 for $n$-heptane mechanism and by a factor of 16.7 for iso-octane mechanism is obtained without losing accuracy of the prediction of autoignition delay times and concentrations of intermediate species.

\section{Introduction}

Improvement of combustion efficiency and minimization of pollutant emissions motivates research on advanced engine concepts such as homogeneous charge compression ignition $(\mathrm{HCCl})$. Understanding of physical and chemical processes involved in the combustion of engine fuels is needed to predict onset of autoignition, oxidation rate, heat release rate, flame extinction, and concentration of species produced during the combustion. The chemical kinetics of fuel oxidation and autoignition plays a critical role in the $\mathrm{HCCl}$ concept.

Detailed chemical kinetic mechanisms have been developed to describe the oxidation of hydrocarbons. These mechanisms are very large in terms of numbers of species and reactions and their size increases with the size of the hydrocarbon. For example, the GRI-mech 3.0 mechanism of methane includes 52 species and 325 reactions [1]. The mechanism of butane elaborated by Pitz and Westbrook at Lawrence Livermore National Laboratory contains 140 species and 807 reactions [2]. In the mechanism of the three isomers of pentane built at the same laboratory, the submechanism of $n$-pentane alone is composed of 193 species and 975 reactions [3]. The mechanism of $n$-heptane worked out by Curran et al. contains 545 species and 2446 reactions [4], and the mechanism of iso-octane by the same authors includes 858 species and 3606 reactions [5]. 
Due to their hierarchical mode of construction, detailed mechanisms of large hydrocarbons contain many species and reactions which are not needed for simulating ignition phenomena over the range of parametric conditions they are intended to cover. These redundant species and reactions needlessly raise the computational time (CPU) of reacting flow simulations that use detailed mechanisms. When large mechanisms are employed, substantial computational resources are required even for zero-dimensional calculations. The size of large mechanisms impacts the time needed to perform reaction-path and sensitivity analyses, which are used to provide valuable insight into processes controlling the ignition of the fuel, the production of pollutant emissions, and the interpretation of experimental results. Reduced mechanisms are needed for use in one-dimensional flame calculations because present solvers are often unable to find solutions when large mechanisms are employed. For example, a reduced mechanism of $n$-heptane deduced from the original mechanism of Curran et al. was used in studies of extinction and autoignition in counter flow configuration which requires a 1-D solver [6].

Practical fuels, such as gasoline and diesel fuels used in automotive engines, are made of hundreds of hydrocarbons belonging to four families: alkanes, alkenes, aromatics, and napthenes. Surrogate fuels composed of a limited number of hydrocarbons representing each family are important in on-going and future research devoted to the emissions of automotive pollutants. The building of the mechanism of a surrogate fuel by gathering detailed mechanisms of individual hydrocarbons will lead to a final mechanism of enormous size, which would require extensive computational resources even for zero-dimensional calculations. In the case of multidimensional simulations in which computational fluid dynamics (CFD), heat transfer, and mass transfer are coupled with chemistry, the use of a detailed mechanism is precluded because CFD calculations alone are already very computationally intensive [7, 8].

For all the reasons given above, it is necessary to use any available technique to produce reduced mechanisms able to reproduce the predictions of detailed mechanisms. A reduction technique has to decrease the number of reactions but mostly the number of species because the memory resources and the computational time increase with the number of differential equations solved by the kinetic code. Indeed, one differential conservation equation is associated with each species. It is well known that large mechanisms always admit some degree of redundancy when 
used for a particular application. Rejecting redundancy is the fundamental principle of a reduction technique.

Tomlin wrote a comprehensive review about reduction techniques applied to combustion mechanisms [8]. Earlier, Griffiths proposed a review dedicated to reduced kinetic models but the purpose of his review is broader and a priori reduced models as the Shell model and the Hu and Keck models are also described [9].

There are many different mathematical methods for reducing detailed mechanisms. However, they can be classified into three families depending on the more or less important chemical character maintained in the description of the mechanism after reduction. (i) The chemical family includes techniques such as the inter-species atomic flow analysis [10, 11], the analysis of reaction and heat release rates [12], the different types of sensitivity analysis $[13,14]$ and their coupling with the principal component analysis [15], the method of directed relation graphs [16-18], the method of directed relation graphs with error propagation [19], an optimization-based approach for obtaining optimally reduced mechanisms by reaction elimination [20] and an associated method for finding rigorous valid ranges for optimally-reduced mechanisms [21]. (ii) The chemico-mathematical family is composed of techniques as quasi-steady state approximation (QSSA) [22, 23], partial equilibrium assumption, computational singular perturbation (CSP) [24], and species life-time analysis [25, 26]. (iii) The mathematical family gathers the repro-modeling technique [27] and the intrinsic low-dimensional manifold (ILDM) technique [28].

Few reduction techniques of the chemical family have been applied so far to a detailed mechanism that includes low $(\mathrm{T}<1000 \mathrm{~K}$ ) and high $(\mathrm{T}>1000 \mathrm{~K})$ temperature combustion chemistry for a large hydrocarbon, to the exception of the analysis of reaction and heat release rates $[29,30]$. We present a new reduction technique, which is able to build automatically the reduced mechanism after detection of unneeded species and reactions. The technique was developed with three aims: (i) to reduce rapidly and with a minimal human intervention large detailed mechanisms, (ii) to build mechanisms for surrogate engine fuels by combining reduced mechanisms of individual hydrocarbons, (iii) to reduce the detailed mechanism of a mixture of hydrocarbon isomers and to extract a submechanism for one component of the mixture. This publication describes how the first aim has been reached.

The reduction technique is presented and its principle is described. Two methods are combined and used to detect redundant reactions. The choice of thresholds 
associated with the two methods and its influence on the size and efficiency of the reduced mechanism is discussed. Finally, the validation of reduced mechanisms for the two reference hydrocarbons: $n$-heptane and iso-octane is presented.

\section{Presentation of the technique}

\subsection{Principle of the reduction technique}

Redundant reactions are recognized by two methods: the analysis of reaction rates and the analysis of sensitivity. The first analysis divides the entire reaction set into two: a group of slow reactions and a group of fast reactions. The analysis of sensitivity also divides the entire reaction set into two: a group of the rate-limiting reactions and a group of the non rate-limiting reactions. The results of both analyses are coupled to identify the redundant reactions. They are non rate-limiting slow reactions. Once the redundant reactions recognized, the redundant species are identified as the species taking part solely in redundant reactions. Finally the reduced mechanism is obtained by removing all redundant reactions and redundant species from the full mechanism.

To predict the chemistry of autoignition of hydrocarbons, the two analyses are performed using the results of a unique simulation of the autoignition of a mixture hydrocarbon $/ \mathrm{O}_{2} /$ diluent in a rapid compression machine or in a shock tube with a detailed mechanism. The simulations are carried out with the chemical kinetic code SENKIN [31] associated with the package CHEMKIN-II [32] by considering an adiabatic and homogeneous system of constant volume.

\subsection{Analysis of reaction rates}

The reaction rate analysis recognizes the fast reactions among the thousands of reactions of the full mechanism. A reversible reaction $i$ is identified as "fast" using its normalized rate of reaction $\overline{r_{\mathrm{i}}(t)}$, which is defined as the absolute value of the ratio of the net rate $r_{i}$ of the reaction (forward rate minus reverse rate) to the highest net rate $\operatorname{Max}\left(r_{\mathrm{i}}(t)\right)$ among all the reaction rates at a time-step $t$. Its value lies between 0 and 1 and takes into account the change of reactivity with time:

$$
\overline{r_{\mathrm{i}}(t)}=\left|\frac{r_{\mathrm{i}}(t)}{\operatorname{Max}\left(r_{\mathrm{i}}(t)\right)}\right|
$$


The group of fast reactions $G_{\text {fast }}$ is selected by the following criterion using the ratethreshold $\varepsilon_{\mathrm{r}}$ :

$$
\text { If } \overline{r_{\mathrm{i}}(t)} \geq \varepsilon_{\mathrm{r}} \text { at each time-step, the reaction belongs to the group } \mathrm{G}_{\text {fast. }}
$$

The analysis is performed automatically by the post-treatment program CKRANA. It calculates the normalized rates over the entire autoignition delay time $t_{\text {ign }}$ and selects the fast reactions according to values of $\varepsilon_{r}$ fixed at $0.1,0.01$, or 0.001 . The fast reactions must be kept in the reduced mechanism to maintain the dynamics of the overall rate of oxidation process. The slow reactions have a normalized rate lower than $\varepsilon_{\mathrm{r}}$ at one time-step at least. They are unimportant in the simulation of the dynamics of the autoignition process and should be discarded. However, some of the slow reactions are bottlenecks for the overall process because no competitive reaction offers a faster pathway. They must be recognized according to a different procedure discussed in the following section and cannot be discarded because of their character of rate-limiting reactions.

\subsection{Sensitivity analysis}

The sensitivity analysis recognizes the rate-limiting reactions of the full mechanism. These reactions can be identified by searching for reactions with high sensitivity relative to a species or a group of species, which plays a leading part in the overall rate of reaction $[8,33,34]$. The radical $\mathrm{OH}$ is a main chain carrier in the oxidation and autoignition chemistry of hydrocarbons and was chosen as the target species. Its rate of production is closely linked to the overall reaction rate during a two-stage autoignition [35]. The program SENKIN calculates the first-order local sensitivity coefficient $S_{\mathrm{j}, \mathrm{i}}$ of the species $j$ and the temperature relative to the reaction $i$ by:

$$
S_{\mathrm{j}, \mathrm{i}}(t)=\frac{\partial Z_{\mathrm{j}}(t)}{\partial A_{\mathrm{i}}}
$$

$Z_{\mathrm{j}}(t)$ is the mass fraction of the species $j$ at time $t$ and $A_{\mathrm{i}}$ is the pre-exponential factor of the reaction $i$. The coefficients $S_{\mathrm{j}, \mathrm{i}}(t)$ are saved in the solution file as the elements of a very large matrix of $N s+1$ rows and $N r$ columns, where $N s$ and $N r$ are the numbers of species and reactions in the full mechanism, and an additional row takes into account the temperature variable. They assess the effect of an absolute infinitesimal change of $A_{\mathrm{i}}$ on the output quantity $Z_{\mathrm{j}}$. The sensitivity coefficients as defined above 
cannot be compared to each other because the parameters $A_{i}$ for reactions of different orders have different units and the values of $Z_{j}$ and $A_{i}$ span many orders of magnitude. The log-normalized sensitivity coefficients $\overline{S_{\mathrm{j}, \mathrm{i}}(t)}$ based on relative infinitesimal changes are preferred:

$$
\overline{S_{\mathrm{j}, \mathrm{i}}(t)}=\frac{\partial Z_{\mathrm{j}}(t) / Z_{\mathrm{j}}(t)}{\partial A_{\mathrm{i}} / A_{\mathrm{i}}}=\frac{\partial \ln Z_{\mathrm{j}}(t)}{\partial \ln A_{\mathrm{i}}}=\frac{A_{\mathrm{i}}}{Z_{\mathrm{j}}(t)} S_{\mathrm{j}, \mathrm{i}}(t)
$$

The post-treatment program CKSENS calculates the log-normalized sensitivity coefficient $\overline{\mathrm{S}_{\mathrm{OH}, \mathrm{i}}}\left(t^{*}\right)$ relative to $\mathrm{OH}$, at a selected time $t^{*}$ and ranks all reactions in decreasing order of sensitivity coefficients according to the index $k$. The reactions are ordered from high sensitivity to low sensitivity. It is necessary to specify a threshold where insensitive reactions can be neglected. As the values of $\overline{S_{\mathrm{OH}, \mathrm{k}}\left(t^{*}\right)}$ cover many order of magnitude, the setting of a reasonable threshold is not an easy task. It is more convenient to define a number of rate-limiting reactions $N_{\mathrm{r}-\mathrm{I}}$. Its value can be obtained by plotting $\log \overline{S_{\mathrm{OH}, \mathrm{k}}\left(t^{\star}\right)}-\log \overline{S_{\mathrm{OH}, \mathrm{k}+1}\left(t^{\star}\right)}$ versus the rank $k$. Figure 1 shows an example of this plot. The prominent maximum observed in this example at a particular rank $k$ corresponds to a large gap between two successive values of $\mathrm{S}_{\mathrm{OH}, \mathrm{k}}\left(t^{*}\right)$. This gap indicates a clear delineation between sensitive reactions and insensitive reactions and is of a great help in defining a sensible value of $k$ as a cutting rank $k_{\text {cut. }}$ It is an indicator of the size order of $N_{r-1}$. The optimal value of $N_{r-1}$ is deduced by testing mechanisms with $N_{\mathrm{r}-\mathrm{l}}$ changed incrementally in steps of 100 . The resulting mechanisms are tested by comparison to the fully detailed model and the optimal mechanism (and $N_{r-1}$ ) is deduced. So, the group of rate-limiting reactions $G_{r-1}$ is selected by the following criterion based on the threshold $N_{\mathrm{r}-1}$ :

If the rank $k$ of the reaction is like $k \leq N_{\mathrm{r}-1}$, the reaction belongs to the group $G_{r-1 \cdot}$

The calculation of the sensitivity coefficients expends large computational resources. A sensitivity calculation using Aurora in Chemkin 4.0 with the iso-octane mechanism of 858 species and 3603 reactions takes 70 hours of computer time on an Intel Zeon 2.2 Ghz processor. A subsequent calculation for a fuel rich case with a 1.2 equivalence ratio did not complete in 200 hours, which was the maximum time limit allowed for runs on this machine. Also the solutions files created by sensitivity 
runs can be very large. A computer run of the SENKIN code of CHEMKIN-II using a methane mechanism of 38 species and 190 reaction produces a solution file of 15 Gigabytes. However, this problem of large solution files has been addressed in later version of CHEMKIN (version 4.0).

Since SENKIN in the CHEMKIN-II suite of codes was used in the present calculations, an alternative strategy had to be undertaken to avoid the expenditure of large computer resources. Several tests indicated that identification of the ratelimiting reactions can be performed at a single time $t^{*}$ and this identification is relatively insensitive to the value of the time $t^{\star}$. For this local sensitivity calculation, it is important to choose a time where the characteristic autoignition chemistry is very active. Reaction path analysis shows that reactions characteristic of autoignition chemistry are very active at a time $t^{\star}$ equal to half of the autoignition delay time. Therefore, this time was chosen to compute these local sensitivities. The calculation procedure was as follows. A SENKIN calculation without sensitivity analysis was performed from time zero to autoignition and the solution file is saved. This calculation was very fast. Then the SENKIN calculation was restarted at a time slightly below $t^{*}$ using the previously computed solution and sensitivity analysis turned on. This procedure saved computing time and memory storage because the calculation of the sensitivity matrix was limited to a few time steps until the time $t^{*}$ was reached. The solution file from the second SENKIN run was used for identification of a group of rate-limiting reactions. We have tested the validity of this procedure on a mechanism of hydrogen. We have compared the values of local sensitivity coefficients obtained with this procedure at selected times when the autoignition chemistry is very active. Then we compared the results to the global sensitivity results computed from a sensitivity analysis calculation starting at time zero. The absolute values of the sensitivity coefficients were not exactly the same in the two cases. However, the relative rankings were exactly the same. As the selection of the group of rate-limiting reactions is based on the ranking of sensitivity coefficients and not on the individual values of sensitivity coefficients, this shows the procedure is valid.

\subsection{Selection of redundant reactions}

Figure 2 illustrates the principle of selection of redundant reactions by coupling the analysis of reaction rates with the analysis of sensitivity. The reactions of the reduced mechanism are the union of the fast reactions $G_{\text {fast }}$ and the rate-limiting 
reactions $G_{r-1 .}$. The discarded redundant reactions are slow and non rate-limiting together.

The program CKRANA builds the reduced mechanism automatically. It is written by selecting the species which appear both as fast and as rate-limiting slow reactions and adding the corresponding reactions and kinetic parameters.

\section{The thresholds, size and efficiency of reduced mechanisms}

The size and the efficiency of a reduced mechanism (RM) depends on the threshold for the fast reactions $\varepsilon_{r}$ and for the rate-limiting reactions $N_{r-1}$. The joint analyses of reaction rates and sensitivities are performed on the results of a autoignition simulation. As the oxidation and autoignition chemistry depends strongly upon the temperature at which it occurs, the input temperature or analysis temperature $T_{\text {ana }}$ of the simulation is also a factor that influences the size and efficiency of the final RM. The values of $\varepsilon_{r}, N_{r-1}$, and $T_{\text {ana }}$ were successively changed keeping the two other parameters constant, and the ignition delay times predicted by the RMs were compared with those predicted by the full mechanism (FM). A discrepancy between the values of ignition delay times predicted by the FM and the RM can be high but not detectable from the graph if these values are small compared to the scale of the graph. To have a reliable criterion of efficiency, we define the average error $E$ on the ignition delay time:

$$
E=\frac{\sum_{1}^{N} \frac{t_{\text {ign }}(\mathrm{RM})-t_{\text {ign }}(\mathrm{FM})}{t_{\text {ign }}(\mathrm{FM})}}{N}
$$

where $t_{\text {ign }}(\mathrm{RM})$ is the ignition delay time predicted by the $\mathrm{RM}, t_{\mathrm{ign}}(\mathrm{FM})$ is the ignition delay time predicted by the FM, and $N$ is the number of simulations performed at different temperatures.

\subsection{Full test-mechanism}

The mechanism of oxidation of $n$-heptane developed by Curran et al. [4] was chosen as fully detailed test-mechanism. $n$-Heptane is the first reference fuel for octane rating for spark-ignited engines and its cetane number is close to that of Diesel fuels. A better understanding of its oxidation kinetics is useful to predict autoignition and pollutant formation in engines. Curran's mechanism describes the oxidation chemistry of $\mathrm{C}_{0}$ to $\mathrm{C}_{7}$ species in the low and high temperature ranges and contains 
545 species in 2446 reactions. Figure 3 shows the main types of reactions of $C_{7}$ species of the primary oxidation mechanism of $n$-heptane. The $n$-heptane mechanism has been validated in a large range of conditions of temperature (650-1550 K), of pressure (2.2-42 bar), of equivalence ratio (0.5-2.0), and of dilution (70-98\%). The experimental data used for validation have been obtained in a variable pressure flow reactor (VPFR) [36], a rapid compression machine (RCM) [37, 38], a jet-stirred reactor (JSR) $[39,40]$, and a shock tube $[41,42,43]$. Typical data were the ignition delay times at low and high temperatures (RCM and shock tube studies), and the species concentration profiles (VPFR and JSR studies). The validated detailed mechanism

allowed assessment of the efficiency of the reduced mechanisms (RMs) in a the same large range of parametric conditions and for various types of data.

\subsection{Influence of the threshold $\varepsilon_{r}$}

The influence of $\varepsilon_{r}$ was studied for calculated ignition delay times in the parametric conditions of the RCM [38]: $650-900 \mathrm{~K}, 3.3-4.5 \mathrm{bar}, \phi=1.0$, and a dilution of $78 \%$. The RMs were generated for the state of a mixture compressed in the RCM at $3.7 \mathrm{bar}$ and $T_{\text {ana }}=710 \mathrm{~K}$ a temperature characteristic of the low-temperature range.

Figure 4 illustrates the influence of the threshold $\varepsilon_{r}$ on the efficiency of the RMs. Four values of $\varepsilon_{r}(1,0.1,0.01$, or 0.001$)$ were used to generate four RMs: red270, red278, red290, and red318 containing 270, 278, 290, and 318 species and $500,526,604$, and 788 reactions respectively. The threshold $N_{\mathrm{r}-\mathrm{l}}$ was kept to 500 . As the numbers of species and reactions in the RM increase when $\varepsilon_{r}$ decreases, the agreement between the results of the RM and the full mechanism (FM) is better when $\varepsilon_{r}$ is smaller. The RM red318 produces results in entire agreement with the FM as confirmed by the low value of the average error: $E=0.7 \%$. The RM red290 can be considered as a suitable compromise between the level of reduction and the efficiency: the predictions are in agreement with the $\mathrm{FM}$ with $E=3.9 \%$. The RM red278 reproduces the shape of the curve well, but the delay times are $42.9 \%$ too long on average. The RM red270 failed completely. Obviously a reduction process cannot be based on a rate-limiting reaction criterion only.

Table I presents the species ranked from $\mathrm{C}_{2}$ to $\mathrm{C}_{6}$ which are suppressed from the FM when $\varepsilon_{r}$ is changed. Eighteen species are suppressed when $\varepsilon_{r}$ is changed from 0.001 (red318) to 0.01 (red290). Most of them are $C_{4}$ species: five are species 
pertaining to the oxidation chemistry of sec-butyl radical and two are radicals formed from but-2-ene (c4h8oh-2, o2c4h8oh-2). Twelve species are suppressed when $\varepsilon_{r}$ is changed from 0.01 (red290) to 0.1 (red278). Again, most of them are $C_{4}$ species: but2-ene, sec-butyl, sec-butylperoxy, 2-hydroperoxybut-3-yl (c4h8ooh2-3) radicals, and but-2-ol-1-yl and but-2-ol-1-peroxy radicals formed from but-1-ene. The corresponding change of $E$ from $42.9 \%$ (red290) to $3.9 \%$ (red278) shows that the oxidation chemistry of butenes and the sec-butyl radical takes a major part in the overall oxidation rate of $n$-heptane. The sec-butyl radical is formed by the addition of a hydrogen atom to the but-1-ene issued from the decomposition of 3-hydroperoxyhept-5-yl, heptenyl, and hept-3-yl radicals. But-2-ene is formed either by the reaction of butenyl radical with $\mathrm{HO}_{2}$ and with ethanal or by the decomposition of c4h8ooh2-3. The later results from an isomerization of sec-butylperoxy radical.

\subsection{Influence of the threshold $\boldsymbol{N}_{\mathrm{r}-1}$}

As for the threshold $\varepsilon_{r}$, the influence of the threshold $N_{r-1}$ on calculated ignition delay times was studied under the experimental conditions of the RCM [38]. The analyses were performed with the set of simulation conditions used to examine the influence of $\varepsilon_{r}$. Six values of $N_{r-1}(0,100,200,300,400$, and 500) generated six RMs with 185, 194, 213, 236, 263, and 290 species and 384, 396, 435, 497, 570, and 604 reactions respectively. The value of $\varepsilon_{r}$ was kept equal to 0.01 .

Figure 5 illustrates the influence of $N_{r-1}$ on the efficiency of the RMs. The agreement between the results of the RM and those of the FM is better when $N_{\mathrm{r}-1}$ is increased. Adding rate-limiting reactions to the group of fast reactions lowers $E$ from $28 \%$ to $3 \%$. The discrepancies are higher in the region $700-860 \mathrm{~K}$ of the negative temperature coefficient (NTC) and in the range $860-900 \mathrm{~K}$ with the maximum discrepancy at the end of the NTC region.

When $N_{r-1}$ is decreased from 200 (red185) to 0 (red213), most of the suppressed reactions are the decomposition of ${ }^{\circ} \mathrm{QOOH}$ to an O-heterocycle and $\mathrm{OH}$ (type 4), the addition of $\mathrm{O}_{2}$ to ${ }^{\circ} \mathrm{QOOH}$ (type 7), the isomerization of ${ }^{\circ} \mathrm{O}_{2} \mathrm{QOOH}$ to ketohydroperoxides (type 8), and ketohydroperoxide decomposition (type 9) (see Figure 3). The results of the brute-force sensitivity analysis of rate parameters to ignition delay time made by Curran et al. at 800 and $910 \mathrm{~K}$ show that reaction types 49 are among the most sensitive [4]. Reaction types 4 to 6 have positive sensitivity coefficients, which indicate that an increase of their rate constants decreases the 
overall reactivity, whereas reactions types 7 to 9 have negative sensitivity coefficients. There is thus a strong competition between the chain-branching reaction paths 7-9 leading to decomposition of ketohydroperoxide and the chain-propagation paths 4-6 of ${ }^{\circ} \mathrm{QOOH}$ radical decomposition, in the NTC region and up to $910 \mathrm{~K}$. The balance between branching and propagation paths is delicate and the suppression of reactions $4,7,8$, and 9 , when $N_{r-1}$ is decreased from 200 to 0 , has a great impact on the overall oxidation rate and thus on ignition delay times. These considerations explain the serious deterioration of the predictions. However, even when $N_{r-1}$ is 0 (i.e. no rate-limiting slow reactions is added to the group of fast reactions), the shape of the curve is retained. By contrast to a reduction process only based on a criterion of the rate-limiting steps, a reduction process only based on the analysis of reaction rates generates quite a satisfactory mechanism.

\subsection{Influence of the analysis temperature $T_{\text {ana }}$}

The influence of the input temperature $T_{\text {ana }}$ of the basic simulation on calculated ignition delay times was studied in the conditions of the shock tube study in [41]: high temperature range $1270-1560 \mathrm{~K}, 2.2$ bar, $\phi=1.0,70 \%$ of dilution in argon. The RMs were generated in the high temperature range for the state of a mixture after the passage of a shock wave at $T_{\text {ana }}=1270 \mathrm{~K}$. The RM red227 with 604 reactions was generated using $\varepsilon_{\mathrm{r}}=0.01$ and $N_{\mathrm{r}=\mathrm{l}}=500$.

In Figure 6, the efficiency of the RM red227 is compared to those of the RMs red290 and red318, which were generated with the conditions $T_{\text {ana }}=710 \mathrm{~K}$, $N_{r-1}=500, \varepsilon_{r}=0.01$ and 0.001 . Figure 6 shows also how the efficiency of the RM changes according to whether $T_{\text {ana }}$ belongs to the low temperature range, here 710 $\mathrm{K}$, or to the high temperature range, here $1270 \mathrm{~K}$. The predictions of the RM red290 are poor with $E=36.4 \%$, whereas the predictions of the RM red227 are very good with $E=2.7 \%$. The reduced accuracy of the RM red290 compared to the RM red227 results from the suppression of key species found in RM red290 inducing a shortening of the delay times. Among them were the radicals $\mathrm{CH}, \mathrm{C}_{2} \mathrm{H}$, allyl, and propargyl, known as essential in the high temperature chemistry. Although the RM red290 has a larger number of species than RM red277, it is less efficient because the analysis temperature $T_{\text {ana }}=710 \mathrm{~K}$ does not belong to the temperature range of the simulated delay times. However, if the level of reduction is lowered by putting $\varepsilon_{r}=0.001$, the corresponding RM red318 reproduces correctly the ignition delay times at high tem- 
peratures with $E=1.7 \%$. Obviously a RM generated with an analysis temperature in the low temperature range is able to predict autoignition in the high temperature range, but a RM generated with an analysis temperature in the high range is not able to predict autoignition in the low temperature range. In fact, all the $\mathrm{C}_{7}{ }^{\circ} \mathrm{O}_{2} \mathrm{QOOH}$ and KETO species of Table II and many similar $C_{6}$ and $C_{5}$ species have been suppressed in the RM red227. At high temperatures the reactions 7, 8, and 9 (Figure 3), in which these species are involved, are slow but non rate-limiting and can be suppressed. At low temperatures they are rate-limiting and their suppression would shut off the low temperature branching and produce a too low rate of oxidation to promote autoignition.

The reduction with an analysis temperature in the high temperature range leads to a higher level of reduction than with an analysis temperature belonging to the low temperature range: 227 species and 540 reactions in the first case against 290 species and 604 reactions in the second case. At high temperature the ${ }^{\circ} \mathrm{QOOH}$ radicals decompose more readily through reactions 4,5 , and 6 instead of adding $\mathrm{O}_{2}$ through reactions 7 (see Figure 3). The contributions of the reactions 7,8 , and 9 to the overall rate of oxidation process become negligible because the decomposition channels are more sensitive to the temperature than the addition reactions 7 which have a zero activation energy. Moreover, the equilibriums $\mathrm{O}_{2}+\mathrm{QOOH}=\mathrm{O}_{2} \mathrm{QOOH}$ shift to the reverse direction in the high temperature range. The reactions 5 are fast and the reactions 4 and 6 are slow and rate-limiting. They are both unaffected by the reduction process. On the other hand, the reactions 7,8 , and 9 are slow but non ratelimiting and can be discarded. The $\mathrm{C}_{7}$ species ${ }^{\circ} \mathrm{O}_{2} \mathrm{QOOH}$ and KETO and some products of decomposition of KETO are suppressed (see Table II) because they are redundant species in the high temperature range, whereas they are essential in the low temperature range. As the symbols ${ }^{\circ} \mathrm{O}_{2} \mathrm{QOOH}$ and KETO stand for multiple isomeric structures, their suppression leads to a drastic reduction of the number of species and reactions. Table II shows that $18 \mathrm{C}_{7}$, seven $\mathrm{C}_{6}$, and six $\mathrm{C}_{5}$ similar species of the types ${ }^{\circ} \mathrm{O}_{2} \mathrm{QOOH}$ and KETO are suppressed in the RM red227. 


\section{Validation of the reduced mechanisms}

\subsection{Reduced mechanism for $n$-heptane}

Table III lists the sizes and parameters of the RMs for $n$-heptane. It also contains the number of rate-determining fast reactions, the number of rate-limiting slow reactions and the gain in CPU time for the simulation of autoignition delay times. The computations were carried out on an IBM Power 4 using 1 processor. The RMs were tested in a large range of conditions (600-1500 K, 3-42 bar, $\phi=0.5-2.0,70 \%-98 \%$ of dilution) and for different types of data (cool flame delay times, ignition delay times, species mole fraction profiles). Simulations of PSR data were performed using the module AURORA of the package CHEMKIN III [44].

Figure 4 shows a comparison between the ignition delay times predicted by the FM and the three RMs red278, red290, and red318 over a range of low temperatures and rather low pressures. The RM red290 offers the best compromise between the level of reduction and the efficiency with $E=3.9 \%$.

Figures 7a-c shows the comparison in a larger range of temperature, at a higher pressure, and for three equivalence ratios. The predictions of red318 are in entire agreement with the FM, the average error $E$ lying between 0.7 and $1.1 \%$. The predictions of the RM red278 are worse below $1000 \mathrm{~K}$ for the lean mixture and in the NTC region for the stoichiometric and rich mixtures, with $E$ between 19.5 and $56.3 \%$. The RM red290 offers the best compromise with $E$ equal to $5.8 \%, 4.5 \%$, and $4.6 \%$ for $\phi=0.5,1.0$, and 2.0 respectively.

Figures $\mathbf{8 a - b}$ and $\mathbf{7 b}$ present the ignition delay times predicted by the FM and the RMs red278, red290, and red318 in the temperature range $650-1340 \mathrm{~K}$ and at $6.5,13.5$ and 42 bar respectively. The RM red318 gives the best agreement with $E$ between 0.8 and $1.3 \%$. The predictions of the RM red278 are worse with $E$ between 21.2 and $43.4 \%$. The RM red290 is the best compromise with $E$ equal to $8.5 \%$ at 6.5 bar, $4.5 \%$ at 13.5 bar, and $4.2 \%$ at 42 bar.

The RMs red278, red290, and red318 were also tested at the higher temperatures of the shock tube study in [41] (see Figure 9). Only the RM red318 gives good results with $E=4.9 \%$. The RM red227 generated at $T_{\text {ana }}=1270$ is more efficient with $E=2.7 \%$, although its number of species is $29 \%$ lower than that of the RM red318. Attempts to reach a higher level of reduction at temperatures above $1290 \mathrm{~K}$ as in 
Coats' shock tube study [42] have shown that the limit in reduction in the high temperature range is reached with the RM red227.

Figure 10 exhibits the comparison between the predictions of the FM and those of the RMs red278, red290, and red318 for mole fraction profiles of oxidation products in the conditions of the JSR study in $[39,40]$. An average error on profile is calculated similarly to the average error on ignition delay time, taking into account each temperature of the profile. Although the gap between the predictions of the RM red278 and the FM looks small, the average error lies between 13.3\% for $\mathrm{CO}_{2}$ and $44.2 \%$ for hept-3-ene. The predictions of the RMs red318 and red290 are better with $E$ between 2.1 and $3.5 \%$, depending on the product. The RM red290 offers the best compromise between the level of reduction and the ability to reproduce the results of the FM.

The speedup factor for the selected RMs varies from 4.2 to 50 : it increases non-linearly with the number of species and cannot be increased more than 50 (see Table III). When $\varepsilon_{r}$ is varied from 0.01 to 0.1 , the CPU time reduction increases slightly but the quality of the predictions is strongly damaged. In most of the parametric conditions, the value $\varepsilon_{r}=0.01$ is the best choice: the corresponding RM red290 gives the best compromise between the level of reduction and the ability to reproduce the results of the FM.

\subsection{Reduced mechanism for iso-octane}

iso-Octane is the second reference fuel for octane rating in spark-ignition engines. It is used as neat fuel and as a component of primary reference fuel blends in current investigations of $\mathrm{HCCl}$ engines. A better understanding of its oxidation kinetics would be very valuable for predicting autoignition and pollutant emissions during the combustion process. Curran's mechanism of iso-octane [5] describes the oxidation chemistry of $\mathrm{C}_{0}$ to $\mathrm{C}_{8}$ species in the low and high temperature ranges and contains 858 species and 3606 reactions. It was validated in a wide range of conditions (550-1700 K, 1-45 bar, 0.3-1.5 for $\phi, 70 \%-90 \%$ dilution) against ignition delay times obtained in shock tubes and species concentrations measured in a JSR, VPFRs, and in a CFR engine. This mechanism is also a valuable test-mechanism for the reduction technique because the efficiency of the RMs can be measured in a large range of parametric conditions and for various types of data. 
Table IV presents the main features of the RMs. The optimal value of $N_{\mathrm{r}-\mathrm{l}}$ was found equal to 500 and three values of $\varepsilon_{r}, 0.1,0.01$, and 0.001 , generated the RMs red300, red325, and red392 respectively. The analysis temperature was taken equal to $702 \mathrm{~K}$ in order to produce RMs valid in the LT and HT ranges. The RMs were tested in the broad range of conditions $(690-1300 \mathrm{~K}, 13-45 \mathrm{bar}, \phi=0.5-2.0,78 \%$ dilution in $\mathrm{N}_{2}$ ) corresponding to the shock tube study in [45].

Figures 11a-c present the comparison between the ignition delay times predicted by the FM and the RMs for three equivalence ratio: $\phi=0.5,1.0$, and 2.0 respectively. The predictions of the RM red392 are in perfect agreement with those of the FM: $E$ varies from 0.2 to $0.7 \%$. Although they appear quite good in the figures, the predictions of the RM red300 are in high disagreement with those of the FM: $E$ varies from 10.8 to $39.2 \%$, the largest discrepancies being above $1000 \mathrm{~K}$ for the lowest ignition delay times. The RM red325 is the best compromise with low values of $E$ equal to $0.8 \%, 2.0 \%$, and $3.8 \%$ for $\phi=0.5,1.0$, and 2.0 respectively.

Figures 12a-c depict the comparison between the ignition delay times predicted by the FM and the RMs for three pressures: 13, 35, and 45 bar respectively. The RM red325 corresponds again to the best compromise with $E=3.6 \%, 3.1 \%$, and $2.6 \%$ at 13,35 , and 45 bar respectively. The same conclusion was drawn when the RMs were tested on species and temperature rise profiles in the conditions of the VPFR study in [36] (Figure 13).

The speedup factor increased non-linearly from 7.7 to 33.3 when the number of species decreased from 392 to 300 (Table IV). When $\varepsilon_{\mathrm{r}}$ is increased from 0.01 to 0.1 , the number of species decrease slightly from 325 to 300 , but the quality of predictions is significantly degraded. The RM red325 is the best compromise.

\section{Conclusion}

For the first time, a new reduction technique of kinetic mechanisms of autoignition based on joint analyses of reaction rates and sensitivities has been developed. The reaction rate analysis divides the entire set of reactions into fast and slow reactions by normalizing the rates and defining a rate threshold $\varepsilon_{r}$. The sensitivity analysis divides the entire set of reactions into a group of rate-limiting reactions and a group of non rate-limiting reactions; a rate-limiting reaction is identified as a reaction exhibiting a high local sensitivity coefficient with respect to the radical $\mathrm{OH}$. The criterion of 
distinction between the non rate-limiting and rate-limiting reactions is based on the threshold $N_{r-1}$, which is the number of reactions in the group of rate-limiting reactions. The optimal values of the thresholds $\varepsilon_{r}$ and $N_{r-l}$ are obtained by a try and error procedure and are specific of the considered full mechanism. They are determined from analyses performed on an arbitrary chosen simulation of ignition. For computing technical reasons, sensitivity coefficients at half of ignition delay time are only considered.

The reduced mechanism is comprised of the union of the set of fast reactions and rate-limiting reactions. Reactions that are not "fast reactions" or "rate-limiting reactions" are redundant and not included in the reduced mechanism. Species included only in these redundant reactions are called "redundant species" and are eliminated from the detailed mechanism.

The reduction procedure is carried out by two computer programs: (i) CKSENS performs the sensitivity analysis., (ii) CKRANA performs the analysis of reaction rates and builds the RM. CKSENS and CKRANA are compatible with the software package CHEMKIN-II.

The technique is fast and easy to apply. A thorough knowledge of the detailed mechanism is not required and only two thresholds of reduction have to be chosen. $A$ $\mathrm{RM}$ is generated in less than five minutes. The longest operation is the finding of the cutting rank to deduce the optimal number of rate-limiting reactions. However, the procedure could be automated.

The reduced mechanisms obtained by our technique are efficient. For Curran's $n$-heptane mechanism, the optimal RM corresponds to a speedup factor of 5.9 and a reduction of $47 \%$ of the number of species, whereas for Curran's iso-octane mechanism it corresponds to a speedup factor of 16.7 and a reduction of $62 \%$ of the number of species. The size of the RM and its ability to reproduce the results of the FM depends on two thresholds and on the temperature at which the analyses are performed. The higher the threshold for the reaction rates analysis, the lower is the size of the RM and the higher the average error. The higher the threshold for the sensitivity analysis, the higher is the number of rate limiting reactions and the better are the predictions of the RM in the negative temperature coefficient region. The best number of rate limiting reactions was found equal to 500 for the two Curran's mechanisms. A RM generated at a high temperature has a smaller size and is more efficient in the high temperature range than a RM generated at low temperature. However, it 
is unable to predict autoignition at low temperature. Conversely, a RM generated at a low temperature has a larger size but is efficient at low and high temperature, provided the level of reduction is not too high. It has to be noticed that the RMs are not only able to reproduce autoignition delay times but also species concentration profiles versus temperature obtained in JSR and VPFR studies.

RMs can facilitate the analysis of reaction flow, reaction path, and sensitivity used to interpret experimental results. They are suitably sized tools for onedimensional flame calculations and are a good starting point for further reduction and multidimensional simulations.

The RMs for $n$-heptane and iso-octane are available at the URL http://wwwcms.IInl.gov/combustion/combustion2.html.

\section{Acknowledgments}

The authors acknowledge the computational center IDRIS of CNRS and especially Isabelle Dupays for her help and support. This work was conducted in part under the auspices of the U.S. Department of Energy by University of California, Lawrence Livermore National Laboratory under Contract No. W-7405-Eng-48. 


\section{Bibliography}

1. Smith, G. P.; Golden, D. M.; Frenklach, M.; Moriarty, N. W.; Eiteneer, B.; Goldenberg, M. et al. http://www.me.berkeley.edu/gri_mech/.

2. Wilk, R. D.; Green, R. M.; Pitz, W. J., Westbrook; C. K., Addagarla, S.; Millze, D. L.; Cernansky, N. P. SAE 1990, Paper 900028.

3. Ribaucour, M.; Minetti, R.; Sochet, L. R. Proc Combust Inst 1998, 27, 345-351.

4. Curran, H. J.; Gaffuri, P.; Pitz, W. J.; Westbrook, C. K. Combust Flame 1998, 114, 149-177.

5. Curran, H. J.; Gaffuri, P.; Pitz, W. J.; Westbrook, C. K. Combust. Flame 2002, 129, 253-280.

6. Seiser, R.; Pitsch, K.; Seshhadri, K.; Pitz, W. J.; Curran, H. J. Proc. Combust. Inst. 2000, 28, 2029-2037.

7. Løwås, T.; Amnéus, P.; Mauss, F.; Mastorakos, E. Proc Combust Inst 2002, 29, 1387-1393.

8. Tomlin, A.S.; Turanyi T.; Pilling, M.J. Low-Temperature Combustion and Autoignition, Comprehensive Chemical Kinetics; Compton, R.G.; Hancock, G., Eds., Elsevier 1997; Vol. 35, Chapter 4, 293-437.

9. Griffiths, J. F. Prog Energy Combust Sci 1995, 21, 25-107.

10. Revel, J.; Boettner, J. C.; Cathonnet, M.; Bachman, J. S. J Chim Phys 1994, 91, 365-382.

11. Soyhan, H. S.; Mauss, F. Combust Sci and Tech 2002, 174, 73-91.

12. Wang, H.; Frenklach, M. Combust Flame 1991, 87, 365-370.

13. Turányi, T. J Math Chem 1990, 5, 204-248.

14. Turányi, T. Reliability Engineering and System Safety 1997, 57, 41-48.

15. Vajda, S.; Valko, P.; Turányi, T. Int. J. Chem. Kin. 1985, 17, 55-81.

16. Lu, T.; Law, C. K. Proc Combust Inst 2005, 30, 1333-1341.

17. Zheng, X. L.; Lu, T.; Law, C. K.; Westbrook, C. K.; Curran, H. J. Proc Combust Inst 2005, 30, 1101-1109.

18. Lu, T.; Law, C. K. Combust Flame 2006, 144, 24-36.

19. Pepiot, P.; Pittsh, H. Systematic reduction of large chemical mechanisms, 4th Joint Meeting of the U.S. Sections of the Combustion Institute, Drexel University, March 21-23, 2005. 
20. Bhattacharjee, B.; Schwer, D. A.; Barton, P. I., Green, W. H. Combust Flame 2003, 135, 191-208.

21. Oluwole, O. O.; Bhattacharjee, B.; Tolsma, J. E.; Barton, P. I., Green, W. H. Combust Flame 2006, 146, 348-365.

22. Tomlin, A. S.; Pilling, M. J.; Turányi, T.; Merkin, J. H.; Brindley, J. Combust Flame 1992, 91, 107-130.

23. Wang, W.; Rogg, B. Reduced Kinetics Mechanisms for Application in Combustion Systems, Lecture Notes in Physics M. 15, Springer-Verlag, 1993.

24. Lam, S. H.; Goussis, D. A. Proc Combust Inst 1988, 22, 931-941.

25. Tomlin, A. S.; Pilling, M. J.; Merkin, J. H.; Brindley, J.; Burgess, N.; Gough, A. Ind Eng Chem Res 1995, 34, 3749-3760.

26. Løwås, T.; Nisson, D.; Mauss, F. Proc Combust Inst 2000, 28, 1809-1815.

27. Turányi, T. Comput Chem 1994, 18, 45-54.

28. Mass, U.; Pope, S. B. Combust Flame 1992, 88, 239-264.

29. Conley, J. P.; Kazakov, A.; Dryer, F. L. Third Joint Meeting of the U.S. Sections of the Combustion Institute, Book of Abstracts, PL06, Chicago, March 2003.

30. Zhao, Z.; Conley, J. P.; Kazakov, A.; Frederick, L. D. SAE 2003, Paper 2003-013265.

31. Lutz, A.E.; Kee, R.J.; Miller, J.A. SENKIN: A Fortran Program for Predicting Homogeneous Gas Phase Chemical Kinetics with Sensitivity Analysis, SANDIA Report SAND87-8248 1988.

32. Kee, R.J.; Rupley, F.M.; Miller, J.A. CHEMKIN-II: A Fortran Chemical Kinetics Package for the Analysis of Gas-Phase Chemical Kinetics, SANDIA Report SAND89-8009B 1991.

33. Nowak, U.; Warnatz, J. Progress in Astronautics and Aeronautics 1987, 113, 87103.

34. Ray, W. J. Biochemistry 1983, 22, 4625-4637.

35. Minetti, R.; Ribaucour, M.; Carlier, M.; Fittschen, C.; Sochet, L.R. Combust Flame 1994, 96, 201-211.

36. Callahan, C. V.; Held, T. J.; Dryer, F. L.; Minetti, R.; Ribaucour, M.; Sochet, L.R.; Faravelli, T.; Gaffuri, P.; Ranzi, E. Proc Combust Inst 1996, 26,739-746.

37. Griffiths, J. F.; Halford-Maw, P. A.; Rose, D. J. Combust Flame 1993, 95, 291306. 
38. Minetti, R.; Carlier, M.; Ribaucour, M.; Therssen, E.; Sochet, L. R. Combust and Flame 1995, 102, 298-309.

39. Dagaut, P.; Reuillon, M.; Cathonnet, M. Combust Sci and Tech 1994, 95, 23360.

40. Dagaut, P.; Reuillon, M.; Cathonnet, M. Combust Flame 1995, 101, 132-140.

41. Vermeer, D. J.; Meyer, J. W.; Oppenheim, A. K. Combust Flame 1972, 18, 327336.

42. Coats, C. M.; Williams, A. Proc Combust Inst 1978, 17, 611-621.

43. Ciezki, H. K.; Adomeit, G. Combust Flame 1993, 93, 421-433.

44. Kee, R.J.; Rupley, F.M.; Miller, J.A.; Coltrin, M.E.; Grcar, J.F.; Meeks, E.; Mo.at, H.K.; Lutz, A.E.; Dixon-Lewis, G.; Smooke, M.D.; Warnatz, J.; Evans, G.H.; Larson, R.S.; Mitchell, R.E.; Petzold, L.R.; Reynolds, W.C.; Caracotsios, M.; Stewart, W.E.; Glarborg, P.; Wang, C.; Adigun, O.; Houf, W.G.; Chou, C.P.; Miller, S.F. Chemkin Collection, Release 3.7, Reaction Design, Inc., San Diego, CA 2002.

45. Fieweger, K.; Blumenthal, R.; Adomeit, G. Combust Flame 1997, 109, 599-619. 


\section{Tables}

Table I: Species suppressed in the FM with $\varepsilon_{r}$ changing from 0.001 to 0.01 then from 0.01 to 0.1 .

\begin{tabular}{|c|c|c|c|c|}
\hline \multicolumn{5}{|c|}{ species of the RM red318 $\left(\varepsilon_{r}=0.001\right)$ suppressed in the RM red290 $\left(\varepsilon_{r}=0.01\right)$} \\
\hline $\mathrm{C}_{2}$ & $\mathrm{C}_{3}$ & $\mathrm{C}_{4}$ & $\mathrm{C}_{5}$ & $\mathrm{C}_{6}$ \\
\hline $\begin{array}{c}\mathrm{c} 2 \mathrm{~h} \\
\mathrm{c} 2 \mathrm{~h} 3 \mathrm{o} 1-2 \\
\mathrm{sc} 2 \mathrm{~h} 4 \mathrm{oh}\end{array}$ & $\begin{array}{c}\text { c3h3 } \\
\text { c3h8 } \\
\text { c3h4-a } \\
\text { c3h4-p } \\
\text { ic3h7o } \\
\text { ch3chco } \\
\text { ch2cch2oh }\end{array}$ & $\begin{array}{c}\text { c4h8ooh2-4 } \\
\text { c4h8ooh2-3o2 } \\
\text { c4h8ooh2-4o2 } \\
\text { nc4ket23 } \\
\text { nc4ket24 } \\
\text { c4h8oh-2 } \\
\text { o2c4h8oh-2 } \\
\text { ic4h8 } \\
\text { c4h10 } \\
\text { c3h8coch3-2 }\end{array}$ & $\begin{array}{c}\text { ic5h9 } \\
\text { bc5h10 } \\
\text { cc5h10 } \\
\text { bc5h11 } \\
\text { dc5h11 } \\
\text { c2h5coc2h5 } \\
\text { c3h6coch3-2 }\end{array}$ & c6h12-3 \\
\hline \multicolumn{5}{|c|}{ species of the RM red290 $\left(\varepsilon_{r}=0.01\right)$ suppressed in the RM red278 $\left(\varepsilon_{r}=0.1\right)$} \\
\hline $\begin{array}{l}\text { o2c2h4oh } \\
\text { pc2h4oh }\end{array}$ & $\begin{array}{c}\text { ic3h7 } \\
\text { ic3h7o2 } \\
\text { ch3coch3 }\end{array}$ & $\begin{array}{c}\text { sc4h9 } \\
\text { sc4h9o2 } \\
\text { c4h8ooh2-3 } \\
\text { c4h8-2 } \\
\text { c4h8oh-1 } \\
\text { o2c4h8oh-1 }\end{array}$ & & nc4h9coch3 \\
\hline
\end{tabular}


Table II: Key species of the RM red290 suppressed in the RM red227.

\begin{tabular}{|c|c|c|c|}
\hline KETO & $\begin{array}{l}\text { nc7ket12 nc7ket13 nc7ket14 } \\
\text { nc7ket15 nc7ket21 nc7ket23 } \\
\text { nc7ket24 nc7ket25 nc7ket26 } \\
\text { nc7ket31 nc7ket32 nc7ket34 } \\
\text { nc7ket35 nc7ket36 nc7ket37 } \\
\text { nc7ket41 nc7ket42 nc7ket43 }\end{array}$ & $\begin{array}{l}\text { nc6ket12 } \\
\text { nc6ket13 } \\
\text { nc6ket14 } \\
\text { nc6ket21 } \\
\text { nc6ket23 } \\
\text { nc6ket24 } \\
\text { nc6ket25 }\end{array}$ & $\begin{array}{l}\text { nc5ket13 } \\
\text { nc5ket14 } \\
\text { nc5ket15 } \\
\text { nc5ket21 } \\
\text { nc5ket24 } \\
\text { nc5ket25 }\end{array}$ \\
\hline${ }^{\circ} \mathrm{O}_{2} \mathrm{QOOH}$ & $\begin{array}{l}\text { c7h14ooh1-2o2 c7h14ooh1-3o2 } \\
\text { c7h14ooh1-4o2 c7h14ooh1-5o2 } \\
\text { c7h14ooh2-1o2 c7h14ooh2-3o2 } \\
\text { c7h14ooh2-4o2 c7h14ooh2-5o2 } \\
\text { c7h14ooh2-6o2 c7h14ooh3-1o2 } \\
\text { c7h14ooh3-2o2 c7h14ooh3-4o2 } \\
\text { c7h14ooh3-5o2 c7h14ooh3-6o2 } \\
\text { c7h14ooh3-7o2 c7h14ooh4-1o2 } \\
\text { c7h14ooh4-2o2 c7h14ooh4-3o2 }\end{array}$ & $\begin{array}{l}\text { c6h12ooh1-2o2 } \\
\text { c6h12ooh1-3o2 } \\
\text { c6h12ooh1-4o2 } \\
\text { c6h12ooh2-1o2 } \\
\text { c6h12ooh2-3o2 } \\
\text { c6h12ooh2-4o2 } \\
\text { c6h12ooh2-5o2 }\end{array}$ & $\begin{array}{l}\text { c5h10ooh1-3o2 } \\
\text { c5h10ooh1-4o2 } \\
\text { c5h10ooh1-5o2 } \\
\text { c5h10ooh2-1o2 } \\
\text { c5h10ooh2-4o2 } \\
\text { c5h10ooh2-5o2 }\end{array}$ \\
\hline $\begin{array}{c}\text { decomposition } \\
\text { products of KETO }\end{array}$ & \multicolumn{3}{|c|}{$\begin{array}{c}\text { nc5h11cho nc5h11co nc4h9cho nc4h9co ch2ch2coch3 } \\
\text { c3h6coch3-1 nc4h9coch2 c2h5coc2h4p c3h6coc2h5-1 } \\
\text { nc3h7coc2h4p }\end{array}$} \\
\hline
\end{tabular}


Table III: Principal features of $n$-heptane mechanisms.

\begin{tabular}{cccccc}
\hline mechanism & full545 & red318 & red290 & red278 & red227 \\
\hline \# species & 545 & 318 & 290 & 278 & 227 \\
\# reactions & 2446 & 788 & 604 & 526 & 540 \\
$\boldsymbol{\varepsilon}_{\mathrm{r}}$ & - & 0.001 & 0.01 & 0.1 & 0.01 \\
$\boldsymbol{N}_{\mathrm{r}-\mathrm{I}}$ & - & 500 & 500 & 500 & 500 \\
$\boldsymbol{T}_{\text {ana }}$ & - & 710 & 710 & 710 & 1270 \\
\# fast reactions & - & 667 & 334 & 109 & 215 \\
\# rate-limiting slow & - & 121 & 270 & 417 & 325 \\
reactions & - & $4.2^{\mathrm{a}}$ & $5.9^{\mathrm{a}}$ & $7.1^{\mathrm{a}}$ & $50^{\mathrm{b}}$ \\
speedup factor & - & & & &
\end{tabular}

a: for the 13 autoignition simulations corresponding to Figure 4

b: for the 13 autoignition simulations corresponding to Figure 9 
Table IV: Principal features of iso-octane mechanisms.

\begin{tabular}{ccccc}
\hline mechanism & full545 & red392 & red325 & red300 \\
\hline \# species & 858 & 392 & 325 & 300 \\
\# reactions & 3606 & 1039 & 731 & 550 \\
$\boldsymbol{\varepsilon}_{\mathrm{r}}$ & - & 0.001 & 0.01 & 0.1 \\
$\boldsymbol{N}_{\mathrm{r}-\mathrm{I}}$ & - & 500 & 500 & 500 \\
$\boldsymbol{T}_{\text {ana }}$ & - & 702 & 702 & 702 \\
speedup factor & - & $7.7^{*}$ & $16.7^{*}$ & $33.3^{*}$ \\
\hline
\end{tabular}

*: for the 11 autoignition simulations corresponding to Figure 11b 


\section{Captions}

Figure 1: Plot of $\log \overline{S_{\mathrm{OH}, \mathrm{k}}\left(t^{\star}\right)}-\log \overline{S_{\mathrm{OH}, \mathrm{k}+1}\left(t^{\star}\right)}$ versus rank $k$ obtained for Curran's mechanism of iso-octane in the following input simulation conditions: mixture iso$\mathrm{C}_{8} \mathrm{H}_{18} / \mathrm{O}_{2} / \mathrm{N}_{2}=0.0165 / 0.2065 / 0.7770$ (in mole fractions), $13.3 \mathrm{bar}, 702 \mathrm{~K}$. In this case the value of $k_{\text {cut }}$ is 420 .

Figure 2: Principle of redundant reaction selection and mechanism reduction

Figure 3: Reaction scheme of the primary oxidation mechanism of $n$-heptane involving $\mathrm{C}_{7}$ species. $\mathrm{RH}$ is for $n$-heptane, $\mathrm{R}^{\circ}$ for heptyl radicals, $\mathrm{Q}$ for $\mathrm{C}_{7} \mathrm{H}_{14}$ structures, $\mathrm{Q}^{\prime}$ for $\mathrm{C}_{7} \mathrm{H}_{13}$ structures, $\mathrm{QO}$ for O-heterocycles, KETO for ketohydroperoxides or aldohydroperoxides and $\mathrm{X}^{\circ}$ for ${ }^{\circ} \mathrm{OH}, \mathrm{CH}_{3}^{\circ}, \mathrm{CH}_{3} \mathrm{O}^{\circ}, \mathrm{CH}_{3} \mathrm{O}_{2}^{\circ}, \mathrm{HO}_{2}^{\circ}$, or $\mathrm{H}^{\circ}$.

Figure 4: Ignition delay times of $n$-heptane $/ \mathrm{O}_{2} /$ diluent mixtures versus temperature calculated predicted with the FM and the RMs with $\varepsilon_{\mathrm{r}}=1$ to 0.001 and $N_{\mathrm{r}-1}=500$. The number of species is given in each case. Conditions of the RCM study in [38]: 3.3-4.5 bar, $\phi=1.0$, dilution with $78 \%$ of air.

Figure 5: Ignition delay times of $n$-heptane $/ \mathrm{O}_{2} /$ diluent mixtures versus temperature calculated with the FM and six RMs with $N_{\mathrm{r}-1}=0$ to 500 and $\varepsilon_{\mathrm{r}}=0.01$. The number of species is given in each case. Conditions of the RCM study in [38]: 3.3-4.5 bar, $\phi=1.0$, dilution $=78 \%$.

Figure 6: Ignition delay times of $n$-heptane $/ \mathrm{O}_{2} /$ diluent mixtures versus temperature calculated with the FM and three RMs : $\operatorname{red} 227\left(N_{\mathrm{r}-1}=500, \varepsilon_{\mathrm{r}}=0.01, T_{\text {ana }}=1270 \mathrm{~K}\right)$, $\operatorname{red} 290\left(N_{\mathrm{r}-1}=500, \varepsilon_{\mathrm{r}}=0.01, T_{\text {ana }}=710 \mathrm{~K}\right)$ and $\operatorname{red} 318\left(N_{\mathrm{r}-\mathrm{l}}=500, \varepsilon_{\mathrm{r}}=0.001\right.$, $T_{\text {ana }}=710 \mathrm{~K}$ ). Conditions of the shock tube study in [41]: 2.2 bar, $\phi=1.0$, dilution with $70 \%$ of $\mathrm{Ar}$.

Figure 7: Ignition delay times of $n$-heptane $/ \mathrm{O}_{2} /$ diluent mixtures versus temperature calculated with the FM and three RMs. The number of species is given in each case. Conditions of the shock tube study in [43]: 13.5 bar, dilution with $78 \%$ of $\mathrm{Ar}$, (a) $\phi=0.5$, (b) $\phi=1.0$, (c) $\phi=2.0$. 
Figure 8: Ignition delay times of $n$-heptane $/ \mathrm{O}_{2} /$ diluent mixtures versus temperature calculated with the FM and three RMs. The number of species is given in each case. Conditions of the shock tube study in [43]: $\phi=1.0$, dilution with $78 \%$ of $\operatorname{Ar}$, (a) 6.5 bar, (b) 42.0 bar.

Figure 9: Ignition delay times of $n$-heptane $/ \mathrm{O}_{2} /$ diluent mixtures versus temperature calculated with the FM and three RMs. The number of species is given in each case. Conditions of the shock tube study in [41]: 2.2 bar, $\phi=1.0$, dilution with $70 \%$ of $A r$.

Figure 10: Mole fraction-temperature profiles of some oxidation products calculated with the FM and three RMs. The number of species is given in each case. Conditions of the JSR study in $[39,40]$ : 9.9 bar, residence time $=1 \mathrm{~s}, \phi=1.0$, dilution with $98 \%$ of $\mathrm{Ar}$, (a) $\mathrm{CO}$ and $\mathrm{CO}_{2}$, (b) heptenes, (c) aldehydes, (d) O-heterocycles.

Figure 11: Ignition delay times of iso-octane $/ \mathrm{O}_{2} /$ diluent mixtures versus temperature calculated with the FM and three reduced mechanisms. The number of species is given in each case. Conditions of the shock tube study in [45]: 40 bar, dilution with $78 \%$ of $\mathrm{N}_{2}$, (a) $\phi=0.5$, (b) $\phi=1.0$, (c) $\phi=2.0$.

Figure 12: Ignition delay times of iso-octane $/ \mathrm{O}_{2} /$ diluent mixtures versus temperature calculated with the FM and three RMs. The number of species is given in each case. Conditions of the shock tube study in [38]: $\phi=1.0$, dilution with $78 \%$ of $N_{2}$, (a) $\phi=13$ bar, (b) 35 bar, (c) 45 bar.

Figure 13: Mole fraction-temperature profiles of some oxidation products calculated by the FM and three RMs. The number of species is given in each case. Conditions of the VPFR study in [36]: $12.5 \mathrm{bar}$, residence time $=1.8 \mathrm{~s}, \phi=1.0$, dilution with $99 \%$ of $\mathrm{N}_{2}$, (a) $\mathrm{CO}$ and $\mathrm{O}_{2}$, (b) temperature rise. 
Figure 1:

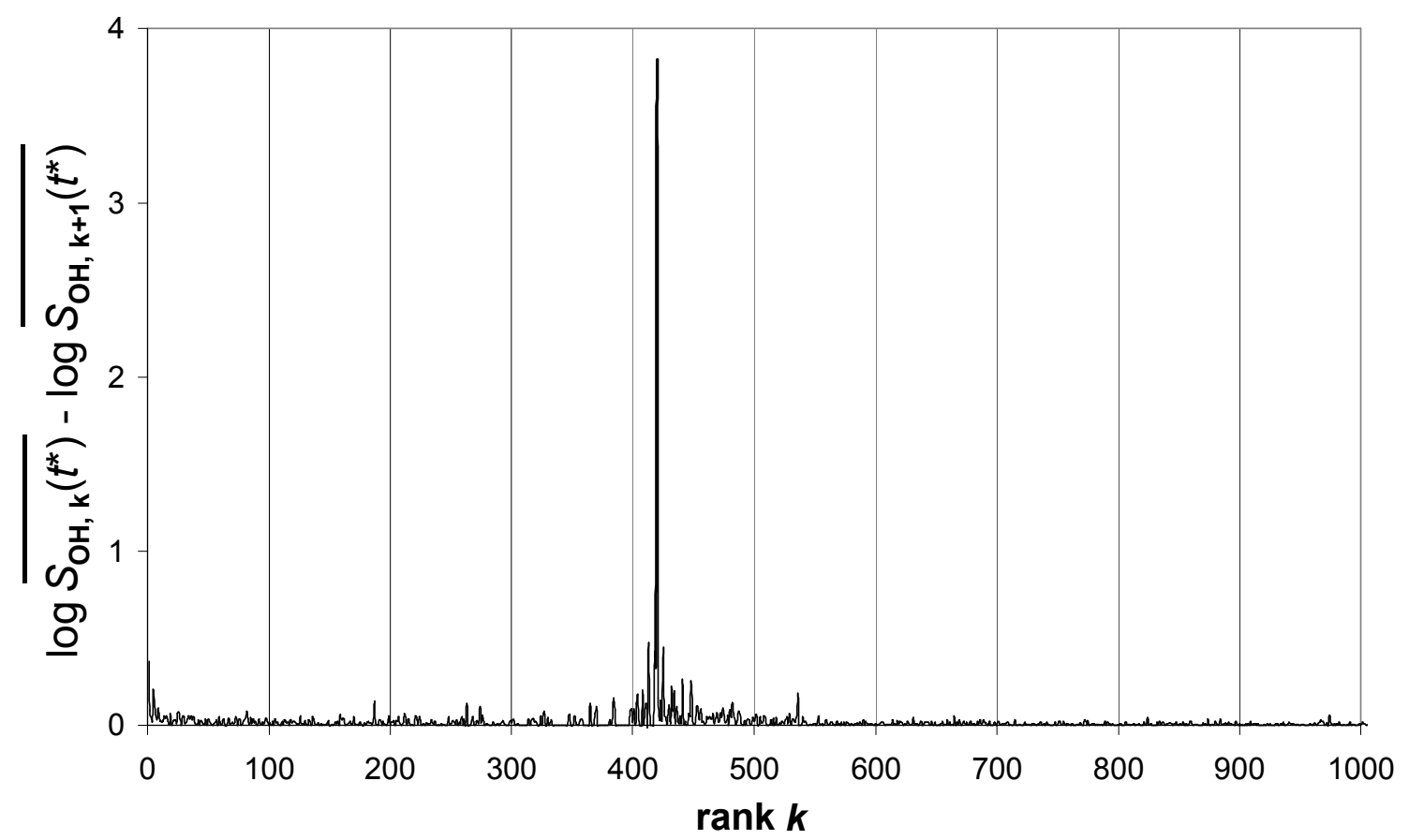


Figure 2:

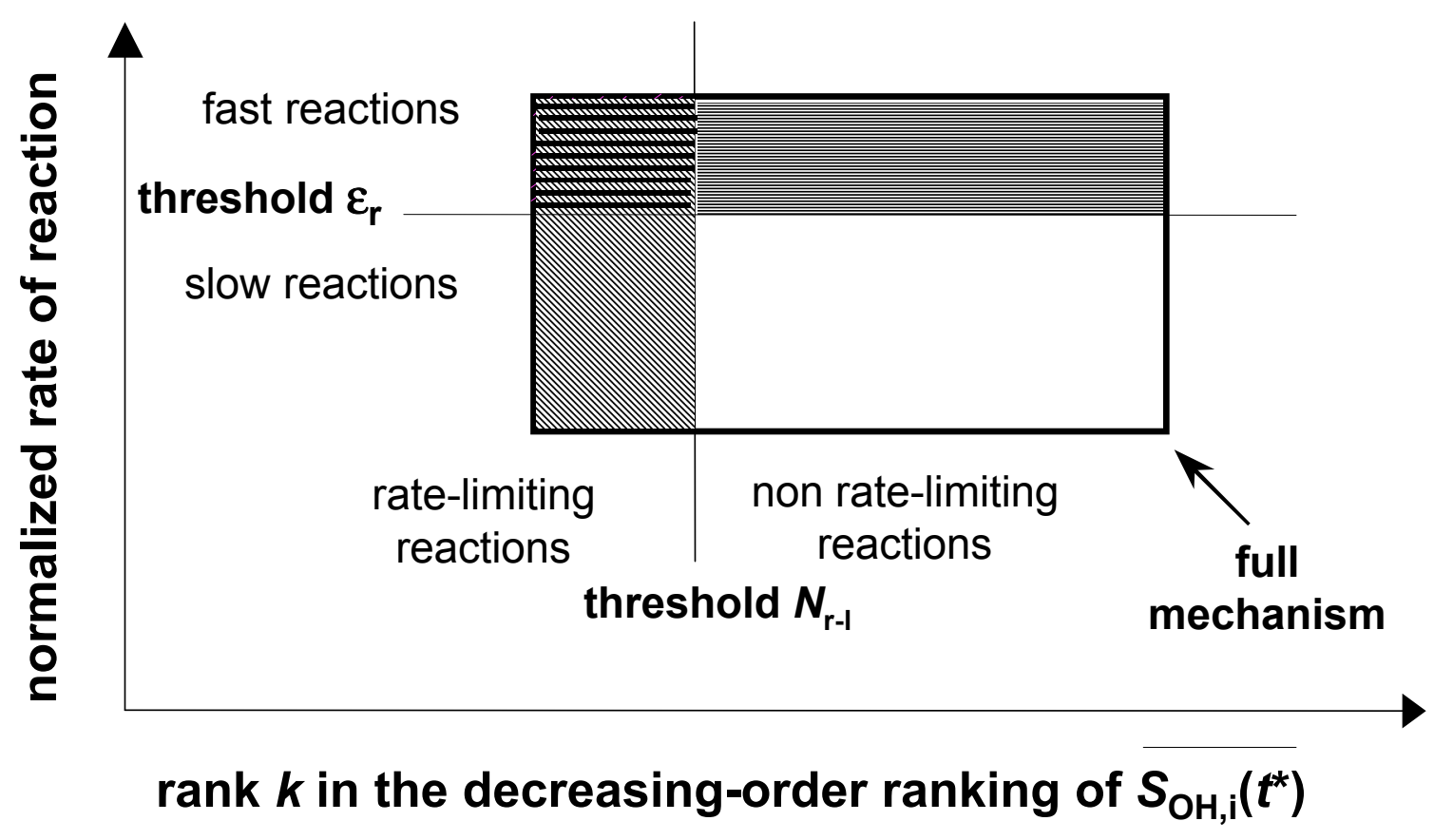

group $G_{\text {fast }}$ of

fast reactions

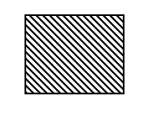

group $G_{r-1}$ of

rate-limiting reactions

redundant

reactions 
Figure 3:

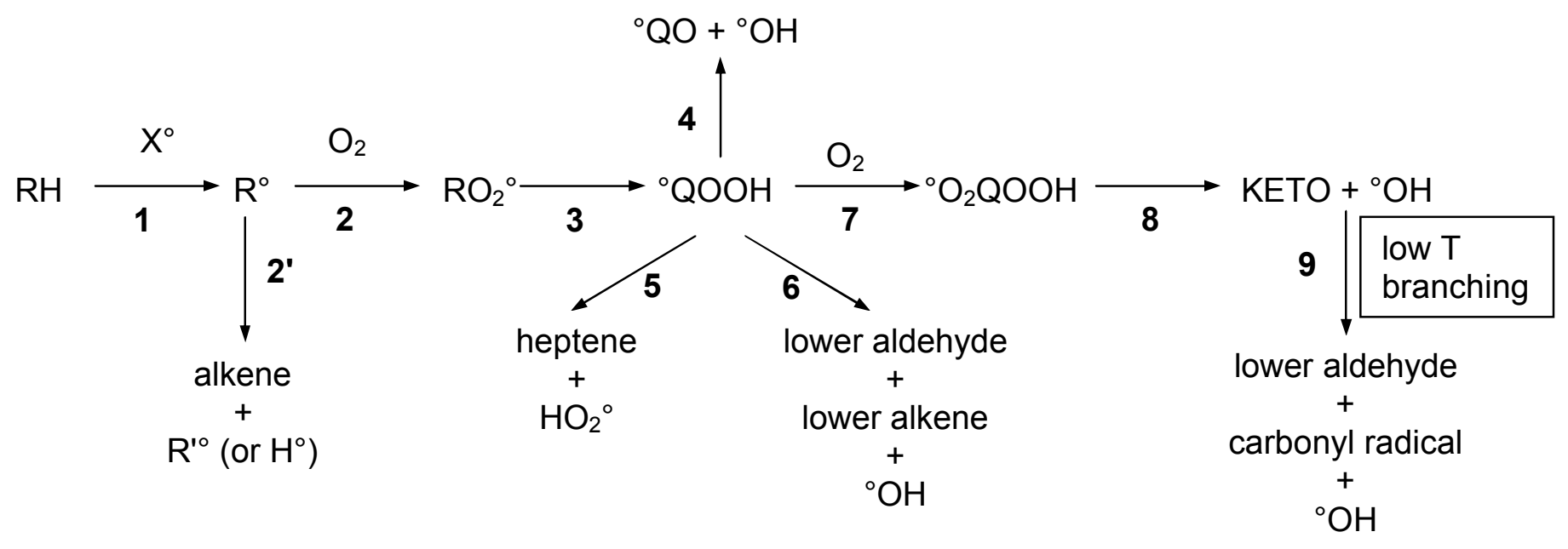


Figure 4:

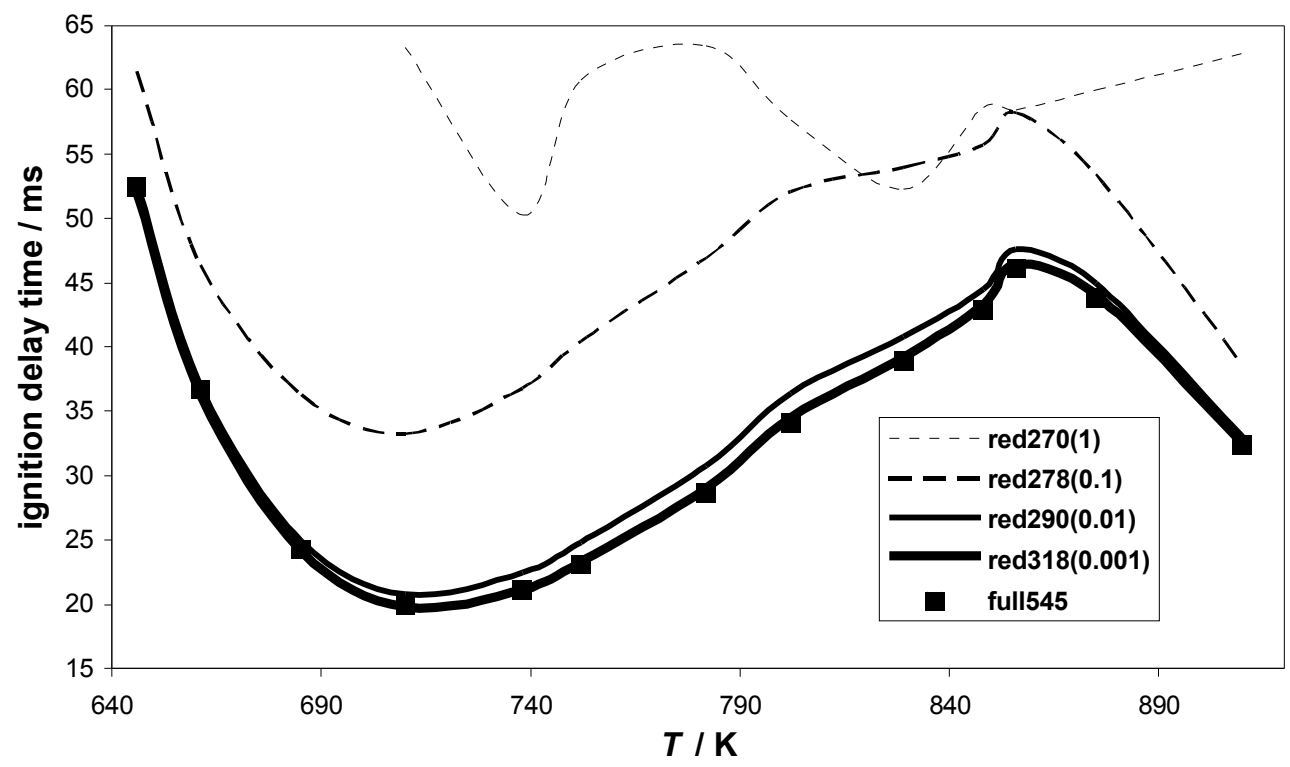




\section{Figure 5:}

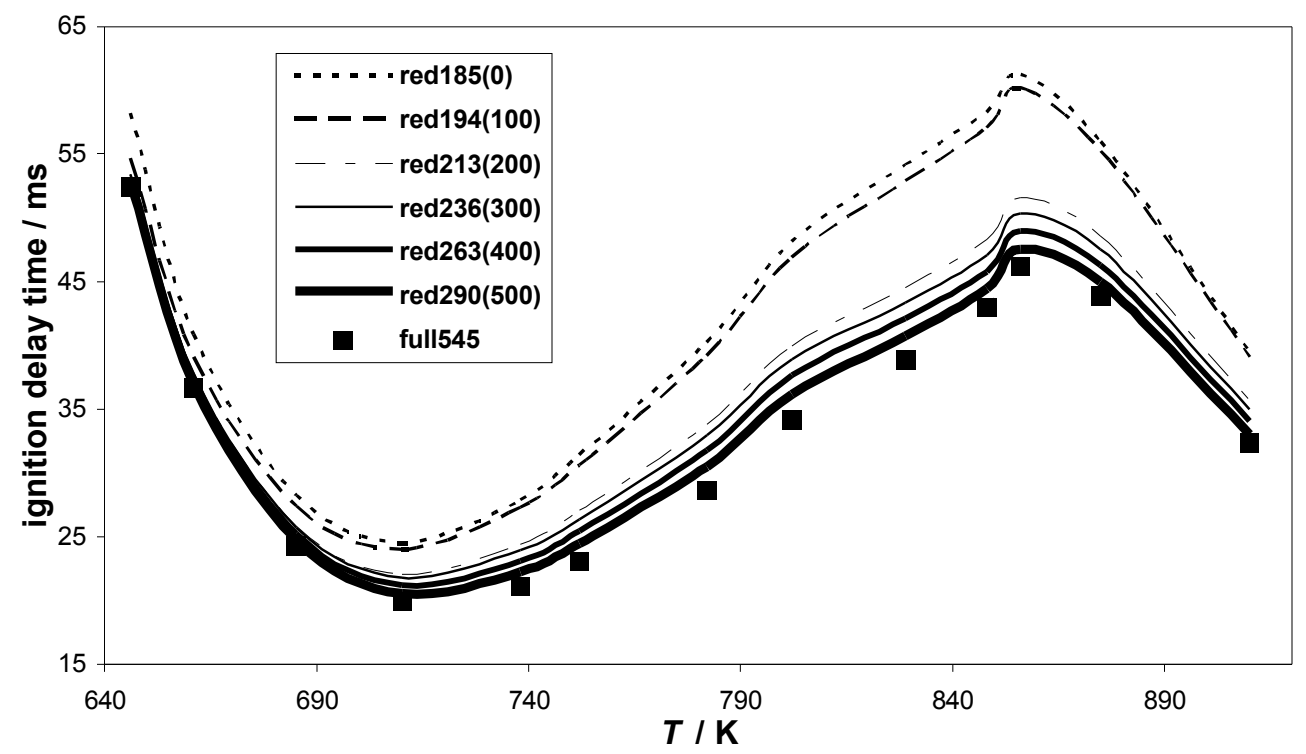


Figure 6:

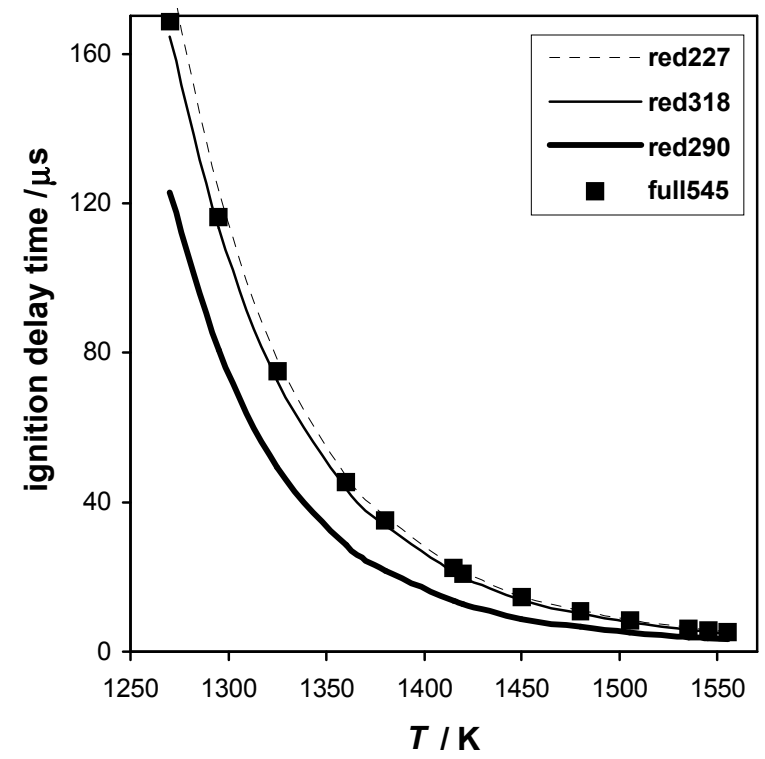


Figure 7:
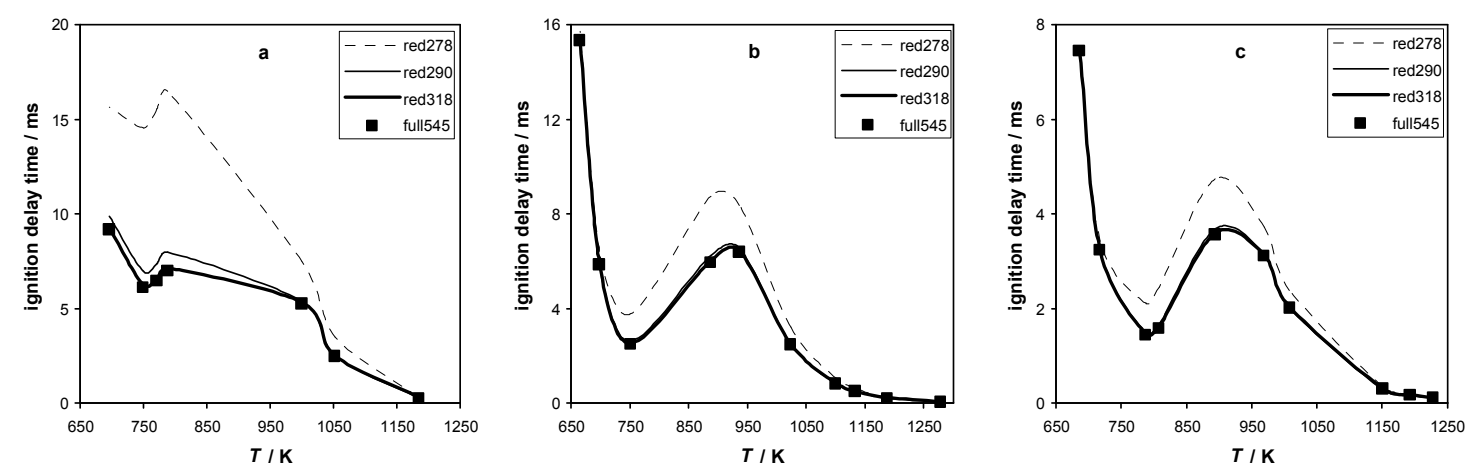
Figure 8:
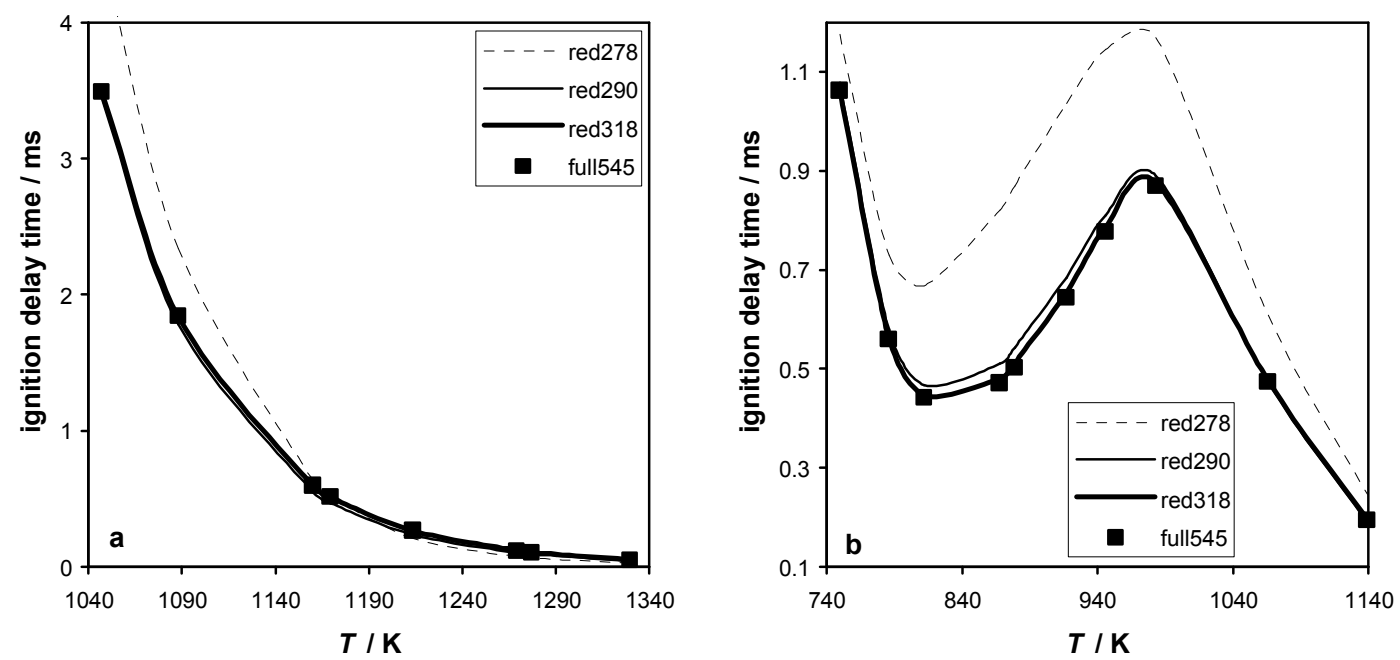
Figure 9:

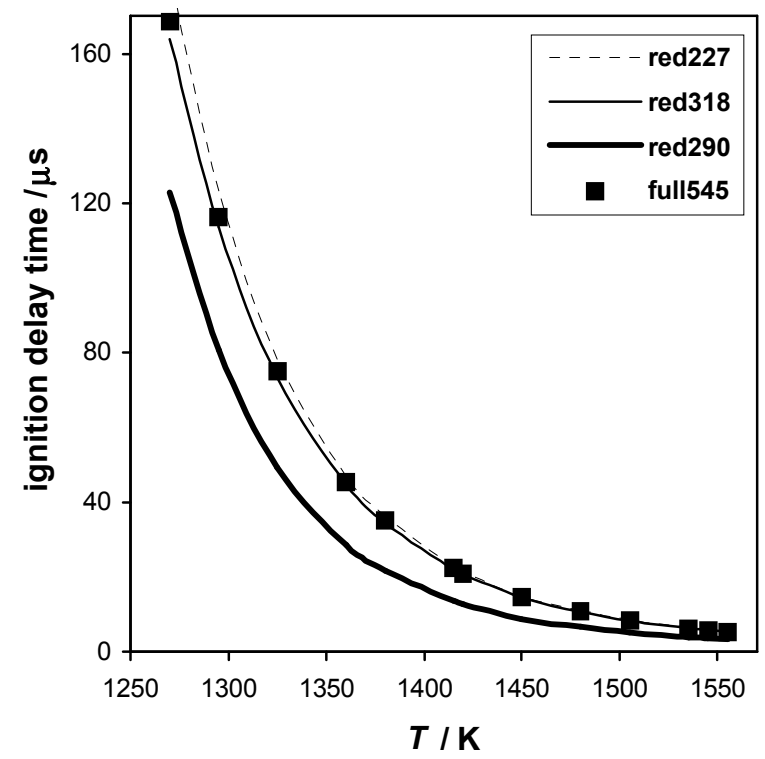


Figure 10:
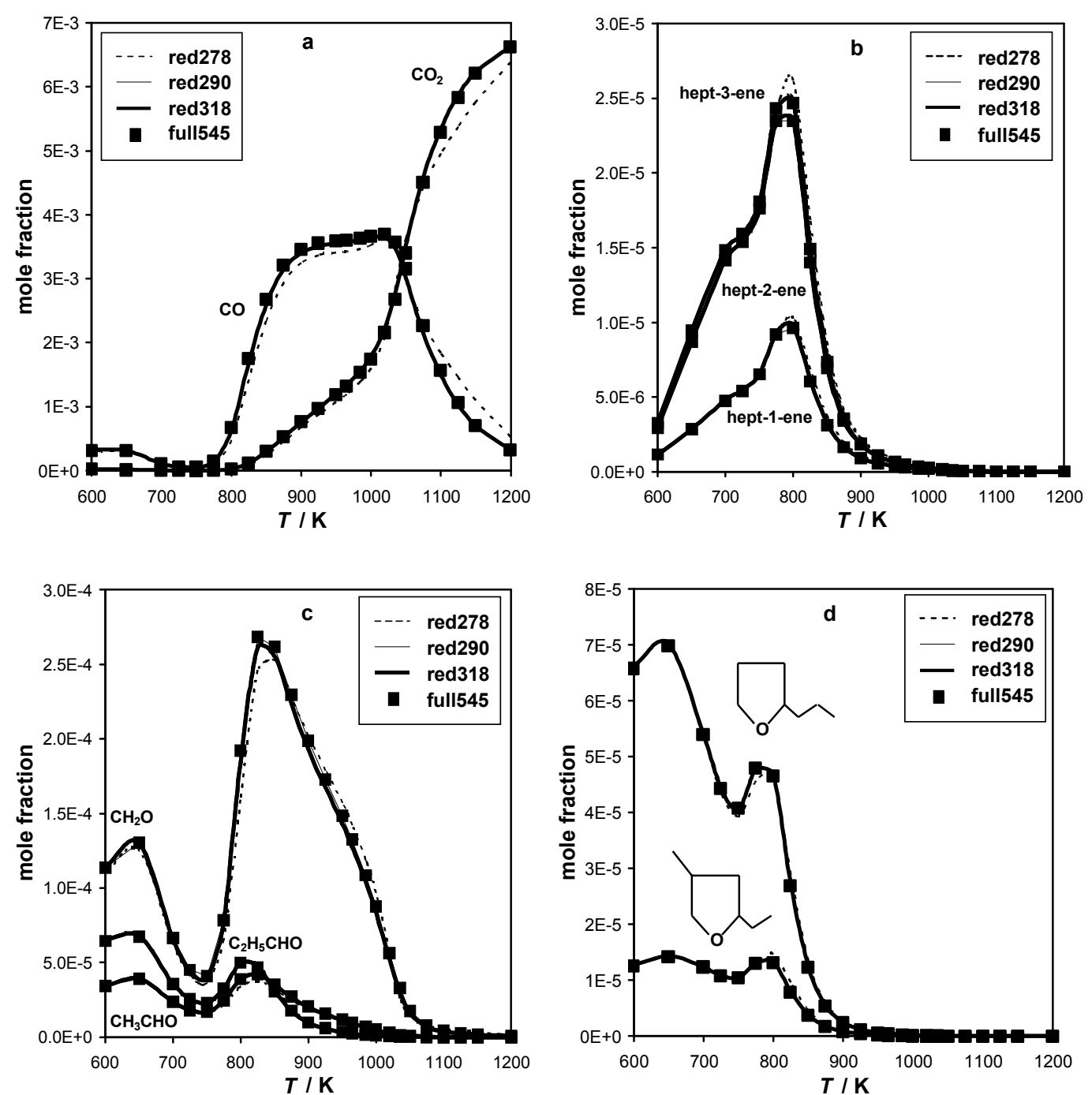
Figure 11:
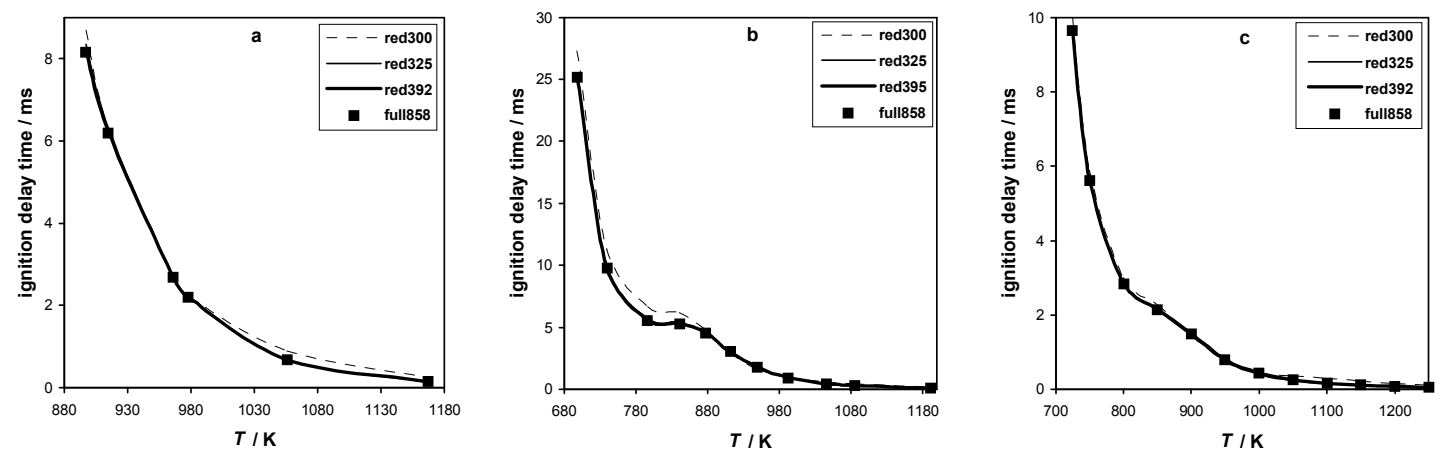
Figure 12:
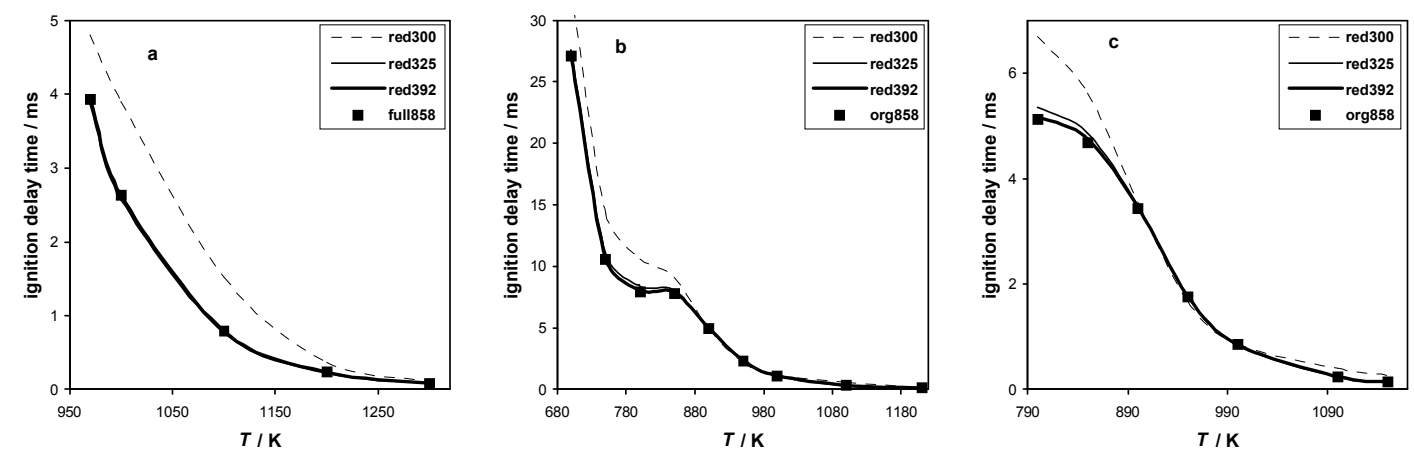
Figure 13:
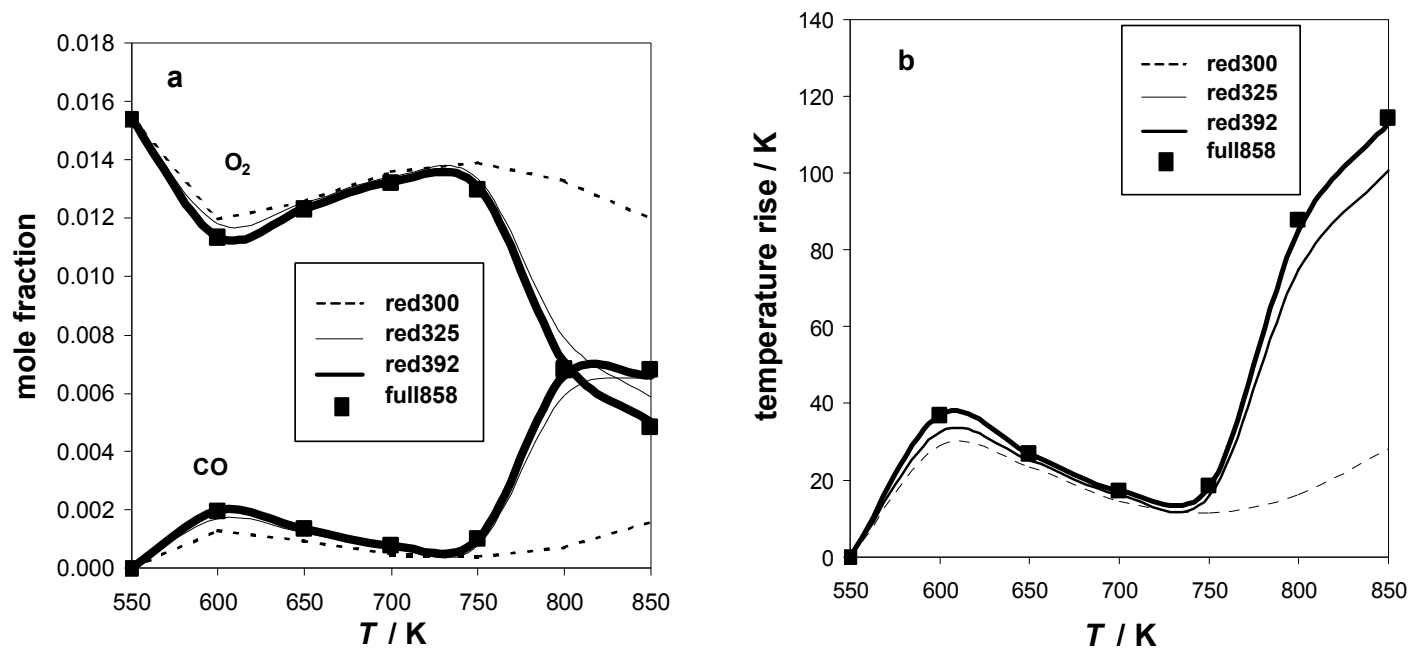